\title{
Cerebellar hemorrhage after resection of frontal meningioma. Case report
}

\author{
Danil Adam ${ }^{1}$, Ioana Hornea ${ }^{2}$, Raisa Croitoru ${ }^{2}$ \\ ${ }^{1}$ Carol Davila University of Medicine and Pharmacy Bucharest \\ 2"Sf. Pantelimon" Emergency Clinical Hospital, Bucharest
}

\begin{abstract}
Introduction: Remote cerebellar hemorrhage is an infrequent complication, potentially harmful with an unclear etiologic mechanism.

There may be incriminated multiple risk factors: arterial hypertension, use of antiplatelet medication (Aspenter), CSF aspiration or drainage associated with intracranial hypotension.

Material and methods: We present a 69 year old woman with remote intracerebral hemorrhage that occurred after frontal parasagittal meningioma resection.

Results: An inadequate oral hydration was responsible for decreased level of consciousness, cerebellar and parietaloccipital cortical-sub-cortical contra-lateral hemorrhages. Close monitoring of water balance and adequate hydration is related to clinical improvement and resolution of brain imaging.

Conclusion: Retraction of the brain due to intracranial hypotension led to some small vessels rupture and development of remote cerebellar hemorrhage.
\end{abstract}

Key words: craniotomy, cerebellar hemorrhage, intracranial hypotension.

Abreviations: $\mathrm{CSF}=$ cerebrospinal fluid; $\mathrm{SSS}=$ superior sagittal sinus; $\mathrm{RCH}=$ remote cerebellar hemorrage.

\section{Introduction}

Cerebral haemorrhage remote from the site of neurosurgical intervention has been described after both supra- or infratentorial cerebral surgeries and after intradural spinal surgeries. Still, it's incidence has a low rate.

The modern imaging techniques allowed this complication to be recognized more frequently since it can sometimes produce the death of the pacient although clinically having no symptoms or manifesting itself as a decreased level of consciousness in many cases. $(1,2)$

Identifying the risk factors and the physiopathological mechanisms as well as such post-surgery haemorrhage prevention have been the main focus of previous studies.

This is a case of frontal parasagittal meningioma, after which resection cerebellar and parieto-occipital haemorrhage occured. The most important etiologic mechanism of such complication is hereby discussed.

\section{Case report}

A 69-year-old woman having a history of type 2 diabetes, second level hypertension, ischaemic coronary artery disease and hyperthyroidism, has presented a series of two episodes of mutism joined by sound producing. 
She has been treated for diabetes for as long as four years with Siofor, for hypertension with Norvasc and for ischaemic coronary artery disease with Aspenter.

A right intracranial parasagittal 38/36/27 mm frontal space occupying lesion has been diagnosed, intensely vascular, having a mass effect on the falx cerebri and a finger shaped edema in white substance (Figure 1). Angio-RM showed superior sagittal sinus permeability and the lack of any vascular cerebral malformations. (Figure 2)

The preoperative coagulation blood tests (PT, APTT, INR, platelet number) have been within normal limits and the blood glucose level was $153 \mathrm{mg} / \mathrm{dl}$. Ten days after Aspenter treatment has been stopped, resection of entire meningioma took place under general anesthesia, supine body position of the pacient, head rotated left, 15 degrees hyperextension.

The $\mathrm{Y}$ shaped scalp incision allowed a trapeze shaped bony flap, with a big base on the median line. After a semi-circular incision of duramater, a well delimited tumoral lesion located in the posterior third of the superior frontal girus became obvios, adherent to the parasagital dura, lateral wall od SSS and to the falx cerebri. Vessels from the brain were also supplying the tumor.

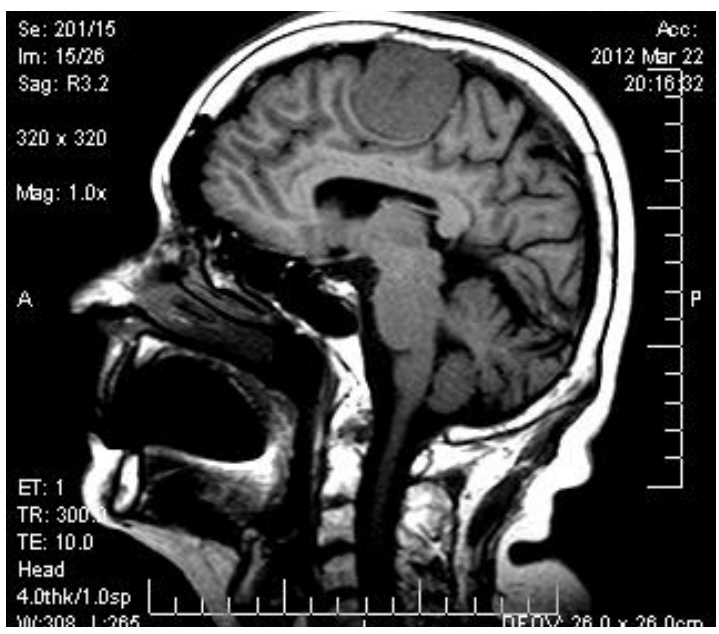

A

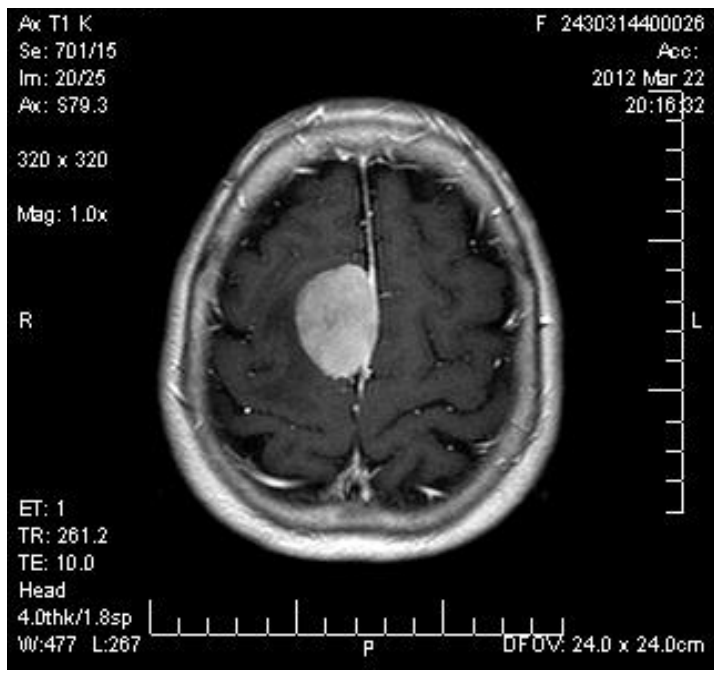

B

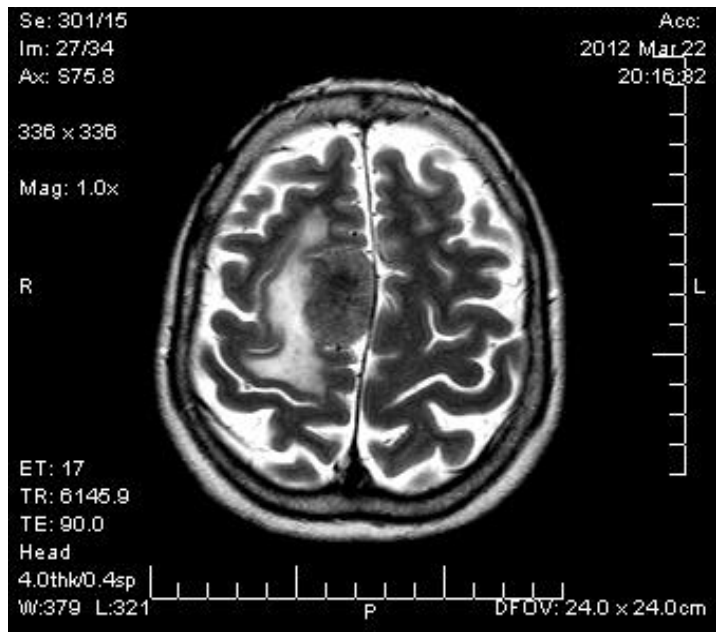

C

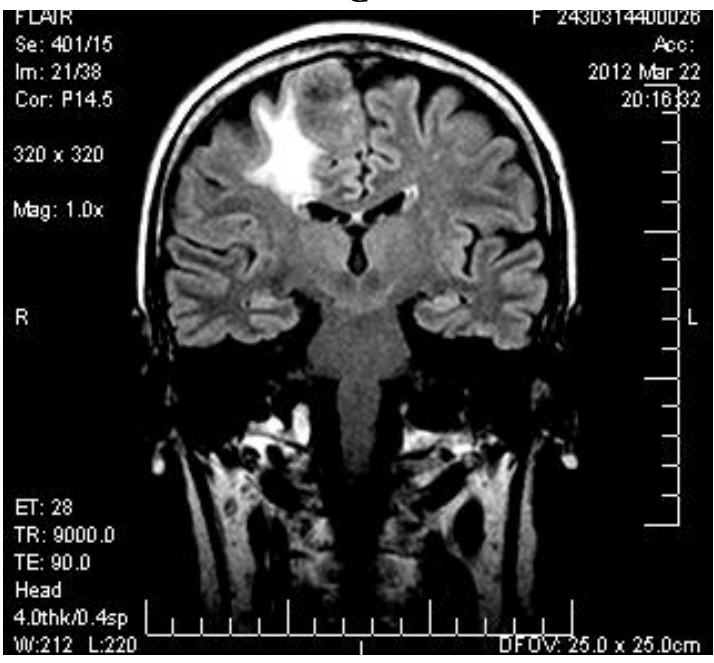

D 


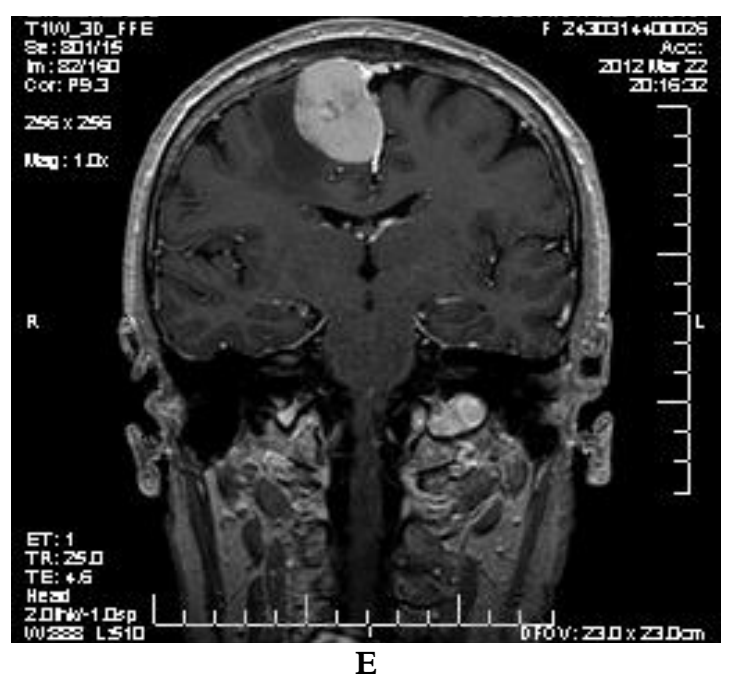

Figure 1. RM pictures of the parasagittal right frontal tumor: sagittal section T1-IRM (A), axial contrast enhanced section T1-IRM (B), axial section T2-IRM (C), coronal section FLAIR-IRM (D), coronal section T1W-3D FFE (E), emphasize the presence of a right frontal parasagittal contrast enhancing tumor with peritumoral edema

The full ablation of the tumor has been undergone together with the coagulation of the insertion base (Simpson grade II resection). The $450 \mathrm{ml}$ intrasurgical blood loss has been compensated with both transfusions of eritrocitary mass and concentrated fresh plasma.

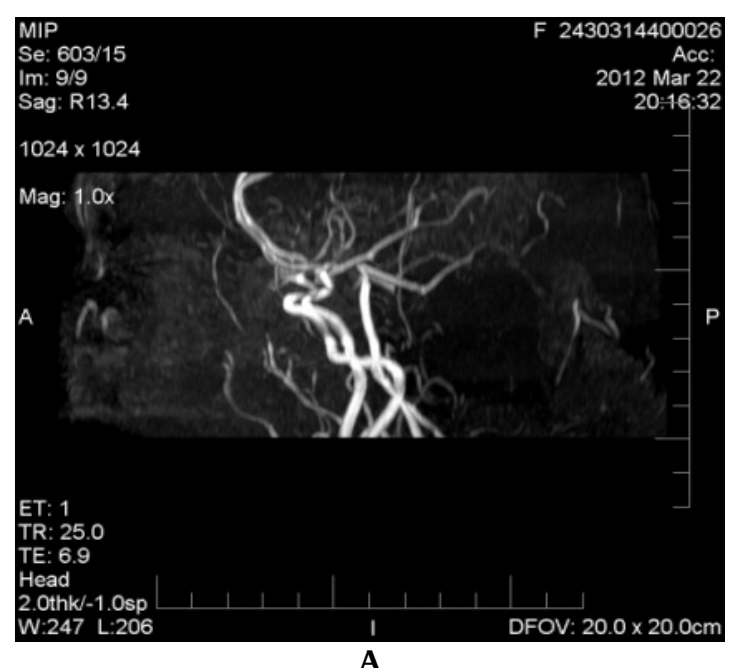

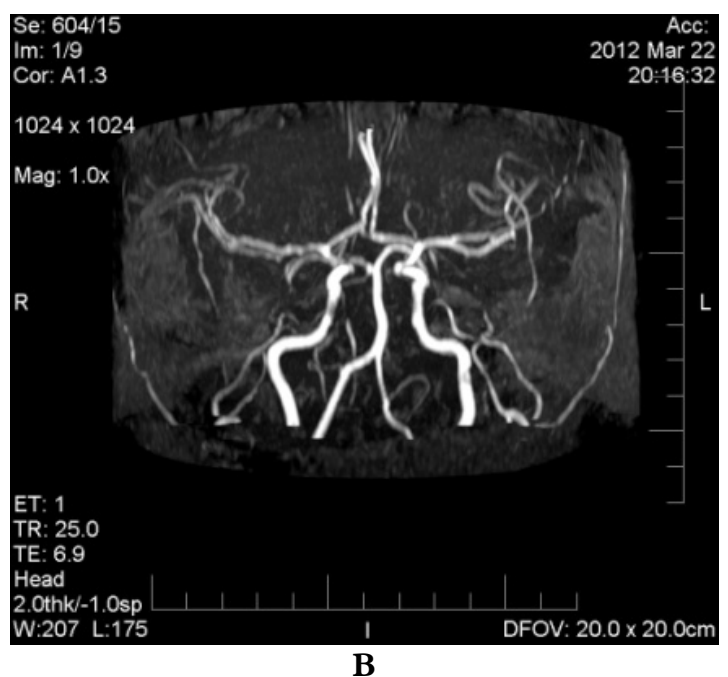

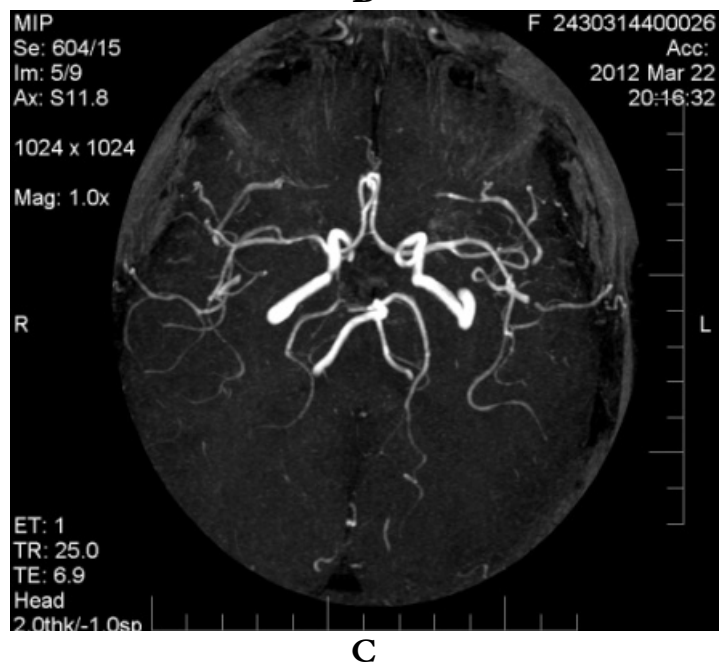

Figure 2. Cerebral angio-RM: sagittal (A), coronal

(B) and axial (C) plans show no vascular malformation

The systolic blood presure oscillated between 134 and $233 \mathrm{~mm} \mathrm{Hg}$ before surgery and between 102 and $119 \mathrm{~mm} \mathrm{Hg}$ intraoperatory. The diastolic pressure changed between $61-79 \mathrm{~mm} \mathrm{Hg}$ intraoperatory. The postoperatory level of glucose in the blood stayed around 150 $\mathrm{mg} / \mathrm{dl}$. The ionogram showed values of $\mathrm{Na}$ $(128 \mathrm{mmol} / \mathrm{l})$ and $\mathrm{K}(2.94,3.05,3.41$ $\mathrm{mmol} / \mathrm{l}$ ) below the inferior limit of the normal. 
After 20 hours from the surgery, the conscious, allert patient showing no sign of neurological deficit has been extubated and the epidural drainage has been suppressed.

During the first 24 hours after the surgery the patient drained $700 \mathrm{ml}$ sanguinolent liquid and she produced a $3000 \mathrm{ml}$ diuresis intraoperative and a 3100 $\mathrm{ml}$ diuresis in the next 20 hours. The intraoperative i.v. provision was $1500 \mathrm{ml}$ of physiological serum and $1500 \mathrm{ml}$ of Ringer's solution to which a $1000 \mathrm{ml}$ physiologic serum and a $1000 \mathrm{ml} 10 \%$ glucose serum have been added postoperative.

During the first postoperative day the patient was able to retake enteral nutrition.

After the surgery,the patient has been treated with $2 \mathrm{~g}$ Ceftadizin, 4 doses, 5000UI/day Fragmin, Aspenter 75 mg/day has been added starting the second day after surgery.

During the third postoperative day, the pacient became sleepy but her state of conciousness improved after a physiological serum perfusion had been administered because the oral hydration was below $1000 \mathrm{ml}$.

The CT scan emphasized a $5 \mathrm{~mm}$ thick bifrontal hygroma, a 2,2/0,9 cm intraparenchymal haematic flow into the right cerebellar hemisphere, bilaterally split between the foliols of the cerebellar hemispheres, and it also showed a small intergiral and pericortical left parietooccipittal haematic flow (Figure 3).

During the next days the apathic state and the unwillingness to take action have regularly improved after physiologic serum perfusions. The CT scan undergone a week after surgery showed dimishing of the haemorrages remote from the site of surgery and maintaining the bilateral hygroma as well as the complete tumor resection without bleeding in the operative site (Figure 4).

An important oral hydration can lead to gradual improvement of the state of consciousness. The patient has been released a week after surgery.

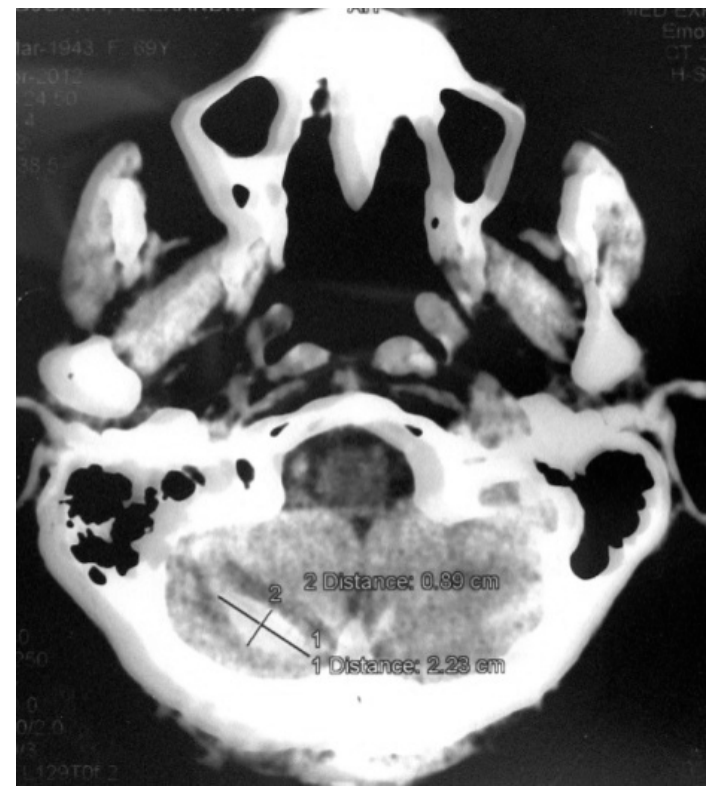

A

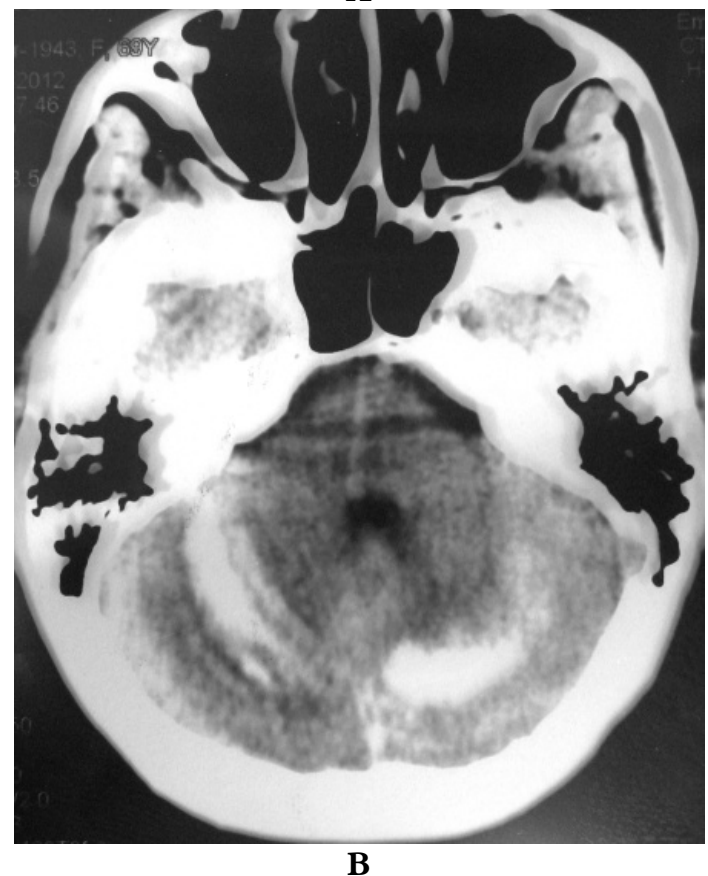



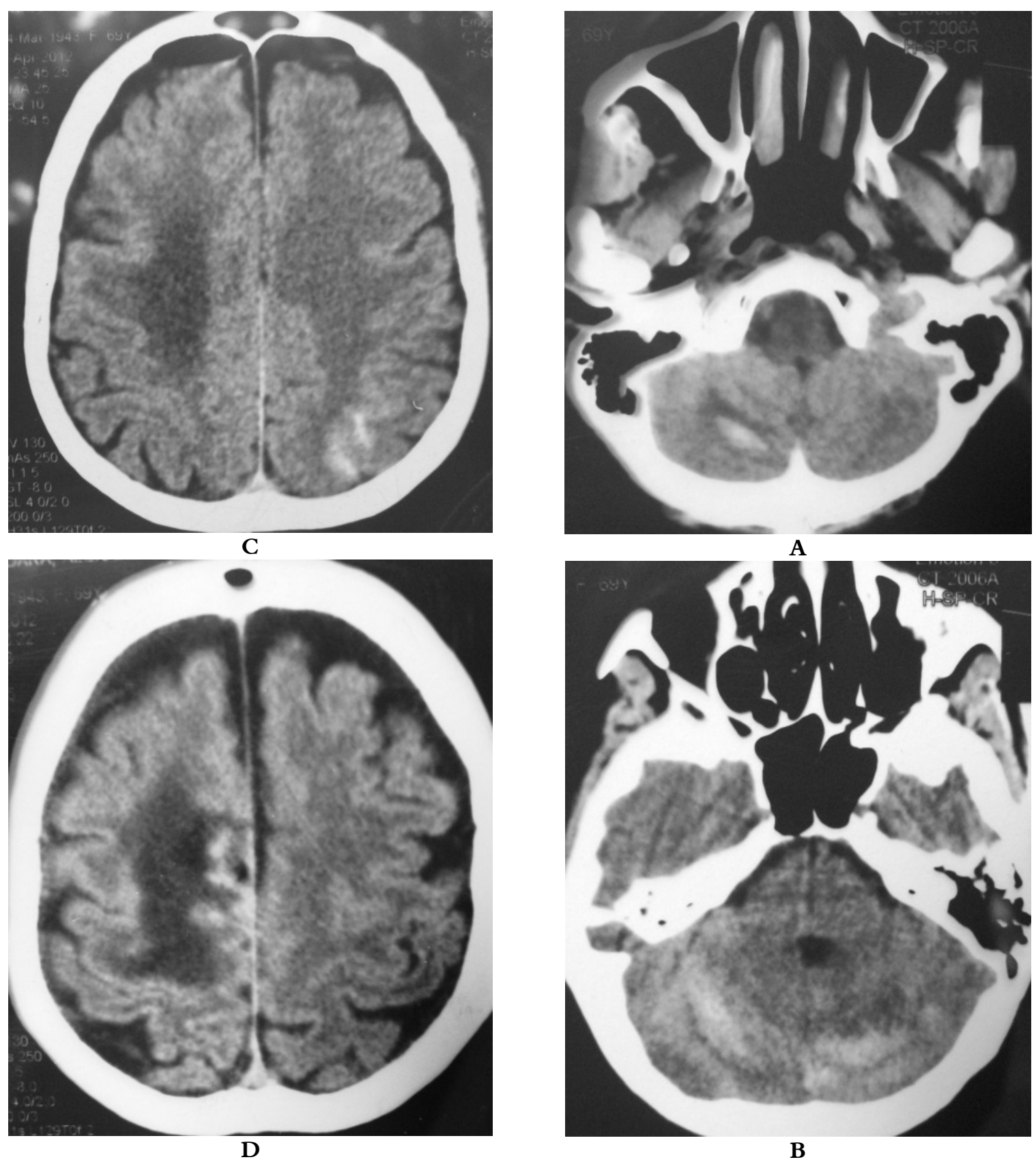

Figure 3. Cerebral CT-scan images: axially, they emphasize bleeding in the right cerebellar parenchyme (A) external of the bilaterral cerebellum's foliage (B), left occipital subcortical haemorrhage (C) and bifrontal hygroma (D) 


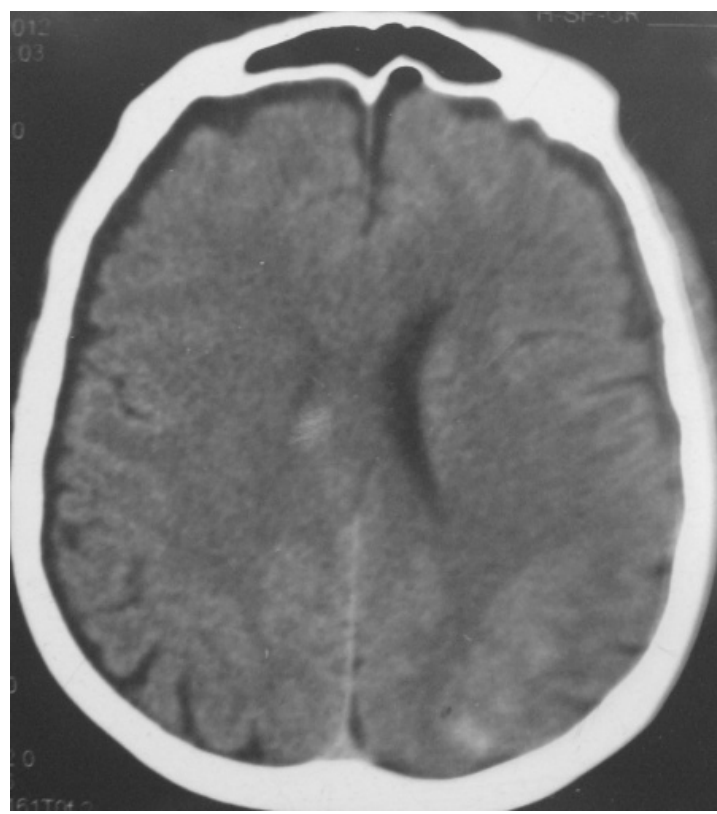

C

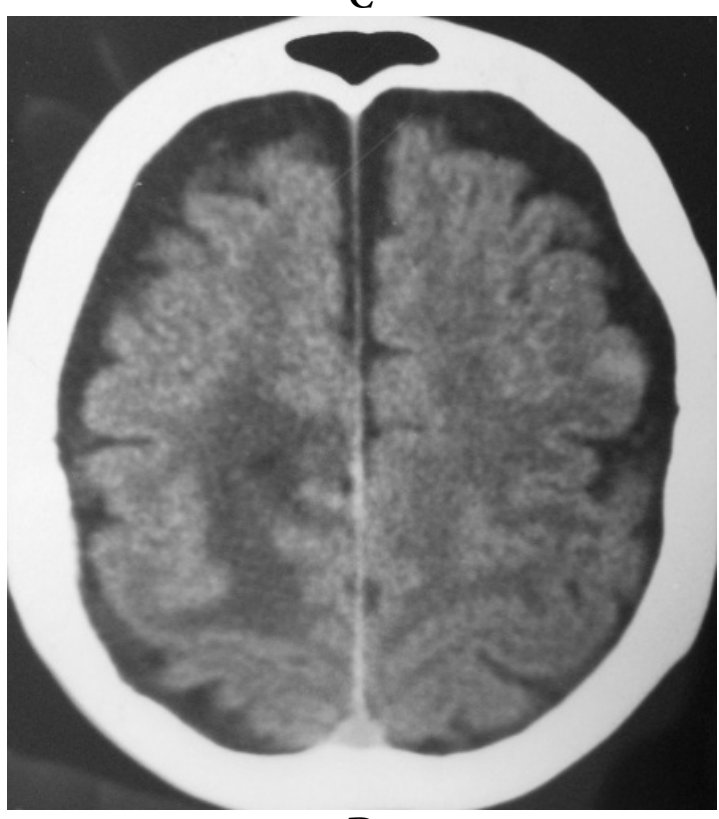

D
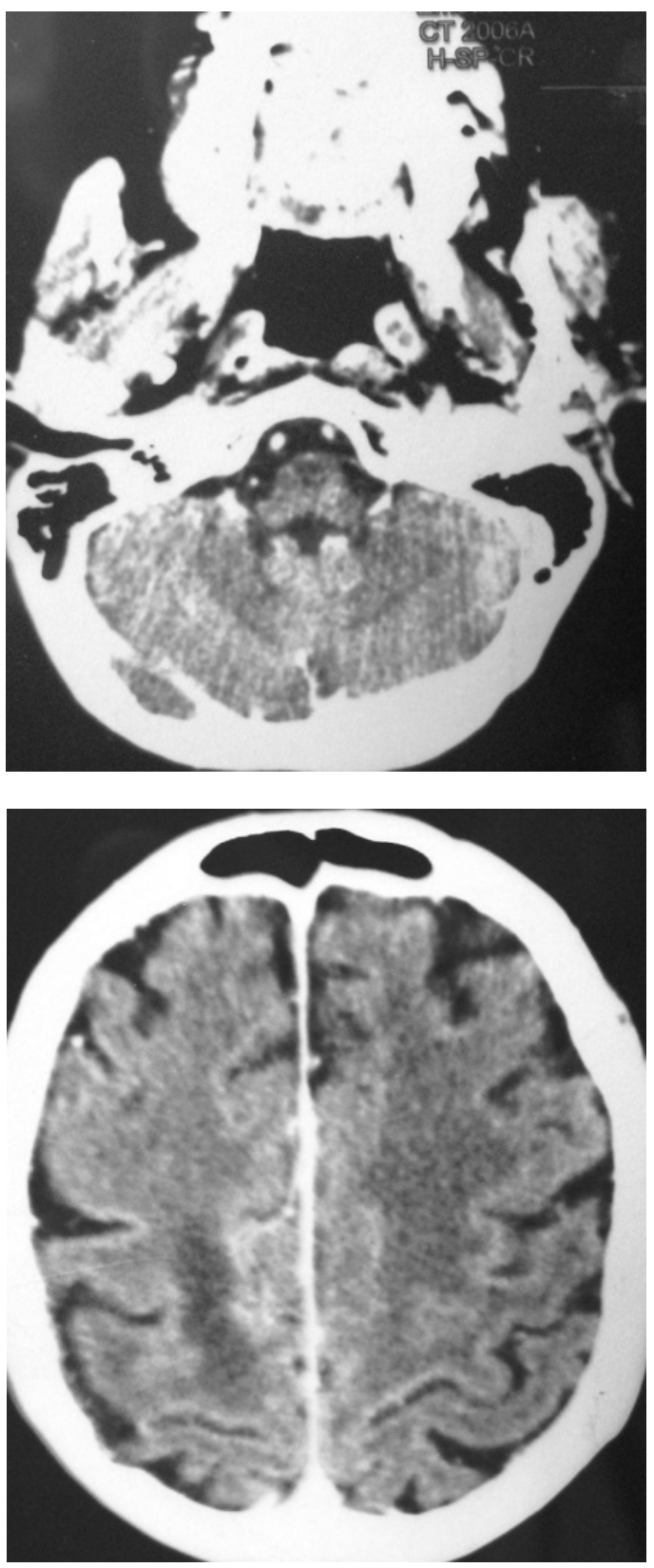

Figure 4. Axial cerebral CT-scan images a week after surgery showing diminishing of the haemorrhages remote from the site of surgery $(\mathrm{A}, \mathrm{B}, \mathrm{C})$ and maintaining of the bilateral hygroma as well as the complete tumor resection without bleeding in the operative focus (D) 


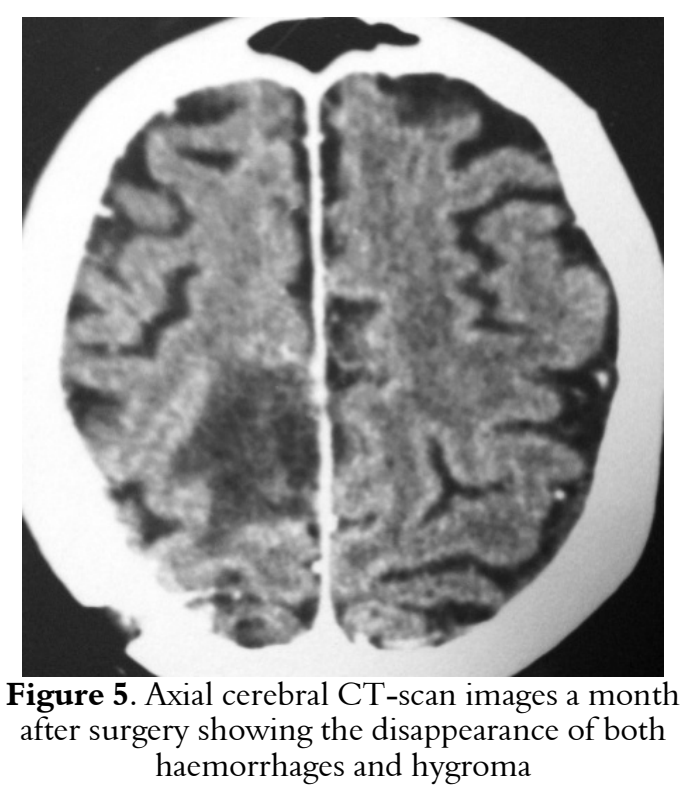

The CT scan undergone one month after surgery shows resolution of both the haemorrhagic lesions and the hygroma and it emphasizes an hipointensive area of $3,5 / 2,8 \mathrm{~cm}$ at the site of surgey (Figure 5).

\section{Discussion}

Posterior cerebral fossa haemorrhage occurring after supratentorial surgery is a rare complication.

The first report of haemorrhage in the pons after a surgery that removes supratentorial lesions has been published in 1937 (3). Such reports have been the result of diagnosis procedures like lumbar puncture and ventriculography. The diagnosis of the haemorrhage was always made postmortem.

Aproximately 60 such cases have been documented until 2002 (5).

Konig and col. (4) have published the first report of a cerebelar haemorrhage complicating a supratentorial craniotomy in 1987.

The reported number of such complications has increased once the computer tomography has been introduced and the neurosurgical comunity became more aware of it's existence.
According to Papanastasiu (2), the rate at which this complication occurs would be 1 in 200 - 400 craniotomies.

Remote cerebellar haemorrhage frequently lacks symptoms which either lead to incidental detection or even the lack of detection.

The most typical appearance in the symptomatic cases is a decreasing level of consciousness (1).

The patient might either be sleepy imediately after extubation or he might become sleepy after being in a state of normal consciousness, depending on the time when bleeding occurs.

The second symptom of the $\mathrm{RCH}$ consists of epileptic crisis which are normally conected with infratentorial haemorrhage lesions placed either in the surgery focus or somewhere remote. In the case we have reported the corticalsubcortical parietal-occipital haemorrhage appeared contralateral to the surgery focus.

Several factors leading to $\mathrm{RCH}$ have been taken into consideration: the arterial hypertension, cerebellar vascular malformations, administering of antiplatelet drugs (aspirine, plavix), position of the patient on the surgery table, CSF drainage.

Several other physiopathological mechanisms have been discussed as playing a part in the development of RCH.

Friedman and col (1) support the venous haemorrhage infarct in the cerebellum, specially around the superior cerebellar vessels which drain the blood from both the vermian cortex and the superior area of the cerebellum to the traverse and torcular sinus. The proof for their point of view consists of many of these lesions' bilateral cerebellar position.

Madow (6) and Poppen (7) consider the venous hypertension as the cause of such complication.

Others think that the loss of a big amount of CSF because of the opening of ventricular cavities, the lumbar drainage or the postsurgical epidural drainage could 
produce the venous haemorrhage infarct and ultimately the RCH (8).

The massive CSF loss could produce the drying of the cerebellum and it's retraction which could generate tension and then closing of bridging vessels as well as higher venous pressure followed by haemorrhage infarct in the area.

Chang and col. (9) think that mentaining of the post surgical drainage produces negative intracranian pressure, retraction of the brain and braking of both little vessels on the surface of the cerebellum and of the capilar bed followed by secondary venous bleeding.

In the case mentioned the retraction of the brain has been documented through postsurgical cerebral CT-scan which emphasized a bilateral hygroma besides the haemorrhage lesions. Still, it wasn't noticeable on the cerebral CT-scan images taken a month after surgery. The change in consciousness has been waving, it got worse during the afternoons but improved after physiological serum and Ringer solution perfusions. This process represented another reason for intracranial hypotension. The case was solved successfully without any surgical treatment.

The literature reports patients that had undergone surgeries for either removing of the cerebellar haematoma or draining of obstructive internal hydrocephalus.

The probability that administration of Aspenter could affect the $\mathrm{RCH}$ is excluded since such treatment has been stopped ten days before surgery.

We also exclude hypertension as one of the favourable disease factors because the sistolic tension has oscillated around 150 $\mathrm{mm} \mathrm{Hg}$.

The supine position of the patient on the surgery table, head rotated left, 15 degrees hyperextension, didn't impact on the venous circulation and can't be considered among the contributing factors in the development of $\mathrm{RCH}$.
A simple measure like the close monitoring of the generated fluid that parallels the diuretic loss can be very useful in RCH prevention. This complication can have important consequences in some cases such as sudden death or longer hospitalization.

In conclusion, considering the reported case, the context within which the $\mathrm{RCH}$ appeared and it's benign development both clinically and imagistically as well as the resolution or the haemorrhage focar areas and of hygroma after close monitoring and hydrating of the patient, both these facts support intracranian hipotension as the main initiatiating factor in the so called "waterfall" of physiopatological mechanisms of $\mathrm{RCH}$ production after supratentorial surgeries.

\section{References}

1. Friedman, J.A., Piepgras, D.G., Duke, D.A., et al Remote cerebellar hemorrhage after supratentorial surgery. Neurosurgery 49, 1327:1340, 2001.

2. Papanastasion, V., Kerr, R., Adams, C. - Contralateral cerebellar hemorrhagic infarction after pterional craniotomy: Report of five cases and review of the literature. Neurosurgery 39(4), 841:852, 1996.

3. Van Gehuchten, P. - Le mécanisme de la mort dans certains cas de tumeur cérébrale. Encéphale 2, 113:127, 1937.

4. König, A., Laas, R., Hermann, H.D. - Cerebellar hemorrhage as a complication after supratentorial craniotomy. Acta neurochir (Wien) 88, 104:108, 1987.

5. Marquardt, G., Setzer, M., Schick, U., Seifert, Y. Cerebellar hemorrhage after supratentorial craniotomy. Surg Neurol 57, 241:251; discussion 251-252, 2002.

6. Madow, L. - Pontine hemorrhage following cranial surgery for supratentorial tumor. Arch Neurol 2, 62:73, 1960.

7. Poppen, J.L., Kendrick, J.F., Hicks, S.P. - Brain stem hemorrhages secondary to supratentorial space-taking lesions. J. Neuropathol Exp Neurol 11, 267:279, 1952. 8. Yoshida, S., Yonekawa, Y., Yamashita, K., et al Cerebellar hemorrhage after supratentorial craniotomy: Report of three cases. Neurol Med Chir (Tokyo) 30, 738:743, 1990.

9. Chang, S.H., Yang, S.H., Son, B.C., et al - Cerebellar hemorrhage after burr hole drainage of supratentorial chronic subdural hematoma. J Korean Neurosurg Soc 46, 592:595, 2009. 